This document is the Accepted Manuscript version of a Published Work that appeared in final form in ACS Applied Materials and Interfaces, copyright @ American Chemical Society after peer review and technical editing by the publisher. To access the final edited and published work see http://pubs.acs.org/articlesonrequest/AOR-eHRzCJhqNRMR7JKA3fAF

\title{
Piezo-Electrochemical Energy Harvesting with Lithium-Intercalating Carbon Fibres
}

Eric Jacques ${ }^{1}$, Göran Lindbergh ${ }^{* 2}$, Dan Zenkert ${ }^{1}$, Simon Leijonmarck ${ }^{2,3}$, Maria Hellqvist Kjell ${ }^{2}$

${ }^{1}$ Department of Aeronautical and Vehicle Engineering, School of Engineering Science, KTH Royal Institute of Technology, SE-100 44 Stockholm, Sweden

${ }^{2}$ Department of Chemical Engineering and Technology, School of Chemical Science and Engineering, KTH - Royal Institute of Technology, SE-100 44 Stockholm, Sweden

${ }^{3}$ Swerea SICOMP AB, Box 104, SE-431 22 Mölndal, Sweden

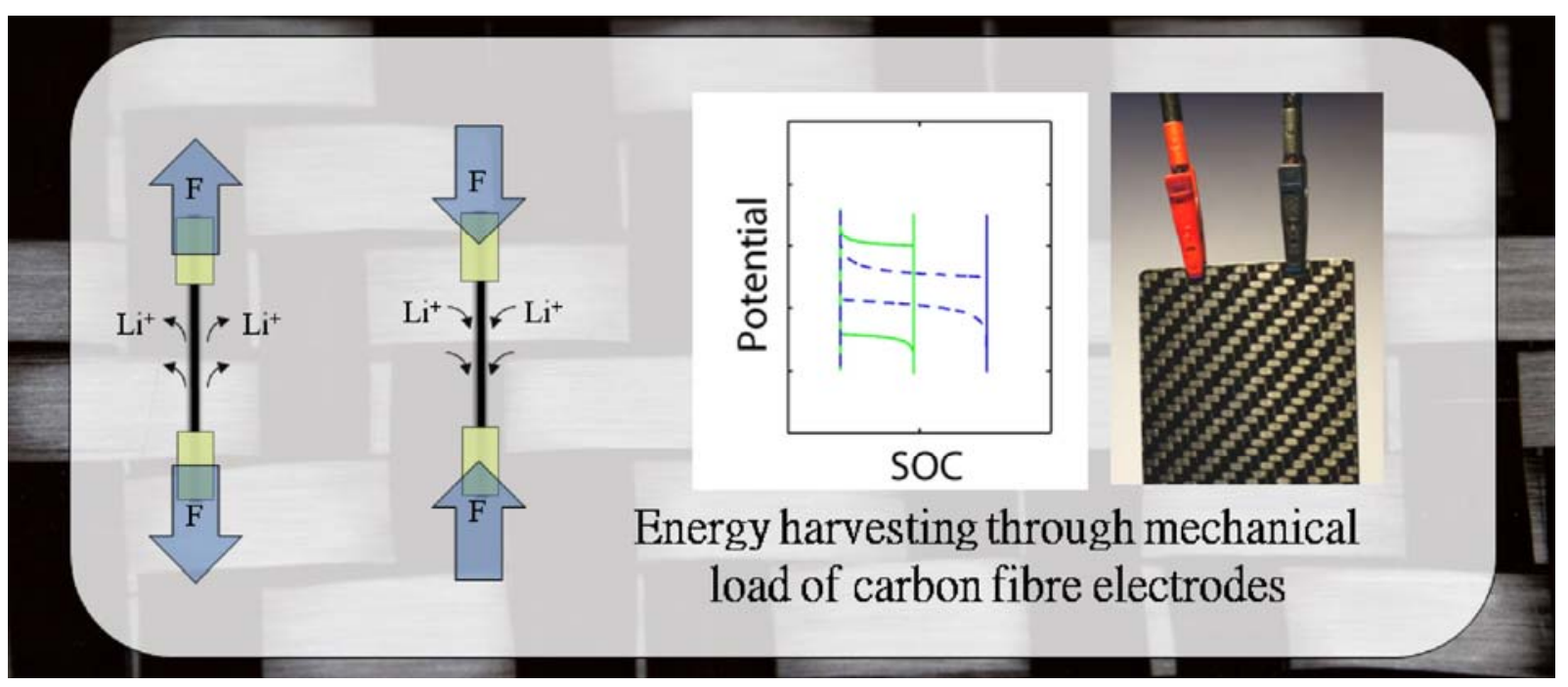

KEYWORDS: carbon fibres, energy harvesting, piezo-electrochemical, lithium-ion intercalation, mechanical energy, electrochemistry 


\section{ABSTRACT}

The mechanical and electrochemical properties are coupled through a piezo-electrochemical effect in Li-intercalated carbon fibres. It is demonstrated that this piezo-electrochemical effect makes it possible to harvest electrical energy from mechanical work. Continuous polyacrylonitrile-based carbon fibres that can work both as electrodes for Li-ion batteries and structural reinforcement for composites materials are used in this study. Applying a tensile force to carbon fibre bundles used as Li-intercalating electrodes results in a response of the electrode potential of a few $\mathrm{mV}$ which allows, at low current densities, lithiation at higher electrode potential than delithiation. More electrical energy is thereby released from the cell at discharge than provided at charge, harvesting energy from the mechanical work of the applied force. The measured harvested specific electrical power is in the order of $1 \mu \mathrm{W} / \mathrm{g}$ for current densities in the order of $1 \mathrm{~mA} / \mathrm{g}$, but this has a potential of being increased significantly.

\section{INTRODUCTION}

In this paper we demonstrate the possibility to harvest electrical energy directly from the mechanical work of a force applied to carbon fibre bundles, by using the fibres simultaneously as an electrode in a Li-ion battery cell. Energy harvesting consists of capturing energy from surrounding sources, converting it and storing it into a usable form. ${ }^{1,2}$ Recent advances in small size and low-power design electronics allowed the use of micro-energy harvesting technologies to move closer toward wireless autonomous electronic devices. ${ }^{3}$ Energy in various forms is harvested from the human body or the environment which can generate $\mu \mathrm{W}$ or $\mathrm{mW}$ level power. ${ }^{1,3,4}$ Microenergy harvesting from kinetic energy (motion or vibrations) is generally the most versatile and ubiquitous. ${ }^{5}$ Kinetic energy is often converted into electrical energy using piezoelectric harvesters that have operating frequencies of typically $100-1,000 \mathrm{~Hz},{ }^{3}$ are small, light and have advantageous energy densities. ${ }^{1,6}$ Lead zirconate titanate (PZT) piezoceramics have high piezoelectric coupling and energy conversion rate, but are too brittle to take general shape. ${ }^{1}$

Rechargeable batteries are the primary electrical energy-storage medium for today's mobile and wearable electrical devices, although with inherent demand of external input in form of contacting and charging. Li-ion batteries are particularly attractive for their high specific energy, high operating voltage and very slow self-discharge, but efforts are made to increase their rate capability. ${ }^{7}$ Intermediate modulus polyacrylonitrile-based (PAN) carbon fibres electrodes exhibit 
promising specific electrochemical storage (Li-intercalating) capacities as battery electrodes. ${ }^{8-10}$ Using carbon fibre electrodes, instead of today's mesocarbon microbeads (MCMB) powder form graphite, allows the electrode to carry mechanical loads. ${ }^{11-13}$ In fact, carbon fibres retain their high stiffness and strength even after large amounts of Li have been intercalated. ${ }^{11,13}$ Recently a piezoelectrochemical effect was found in lithiated carbon fibres. ${ }^{14}$ When lithium ions are intercalated (inserted) into the carbon fibre electrode it swells ${ }^{12}$ causing the fibre to expand both longitudinally and radially. This is a well-known effect in most electrode materials. However, it was also found that the electrical potential of the carbon fibre electrode changes when a mechanical load is applied in a similar way to a piezo-electric material. A clear physical explanation for the potential change due to mechanical loads is still pending. Previous work has used a similar effect in intercalated graphite for electrochemical actuator applications because of the low coupling factor it provides (i.e., high voltage-induced stress, contrary to piezoelectrics which have low voltage-induced strain). ${ }^{15-17}$ The possibility to harvest and store energy in a single device has also been demonstrated using piezo-electric materials as separator in a lithium-ion battery coin cell, where an external alternating mechanical load was used for charging the battery. ${ }^{19,20}$

The aim of this paper is to demonstrate the possibility to harvest energy using lithium intercalated load carrying carbon fibre electrodes. This might be achieved by converting the energy from mechanical work of an external force applied to the Li-intercalated carbon fibre electrode releasing more electrical energy during discharge than provided at charge of the same capacity by using this piezo-eletrochemical effect. Energy harvesters with high strength, flexibility, conceivable internal storage capability and with general shape might thus be obtained using carbon fibre electrodes in a Li-ion battery-like device. ${ }^{18}$

\section{EXPERIMENTAL SECTION}

\section{Materials and test specimen design}

This study was conducted on PAN-based carbon fibre bundles of Torayca T800HB 6K 40B P1 BB 223tex and unsized Toho Tenax IMS65 24K 830tex. These grades are particularly favourable due to their high tensile strength and elastic modulus, and good electrochemical specific capacities to intercalate $\mathrm{Li}$ which can be up to $300-350 \mathrm{mAh} / \mathrm{g}$ at low current densities (charge/discharge current per electrode mass) when the diffusion of $\mathrm{Li}$ in the electrolyte and carbon fibre bundle is not rate limiting. ${ }^{9,10}$ Test specimens made of carbon fibre bundles were designed and manufactured 
for multifunctional experiments, i.e., for use in strain-controlled mechanical tests and as electrode in Li-ion cells simultaneously, as shown in Figure 1. The IMS65 bundle was split into a lighter one of about 12,600 filaments (estimated by weighing) to ease the diffusion of $\mathrm{Li}$ in the bundle).
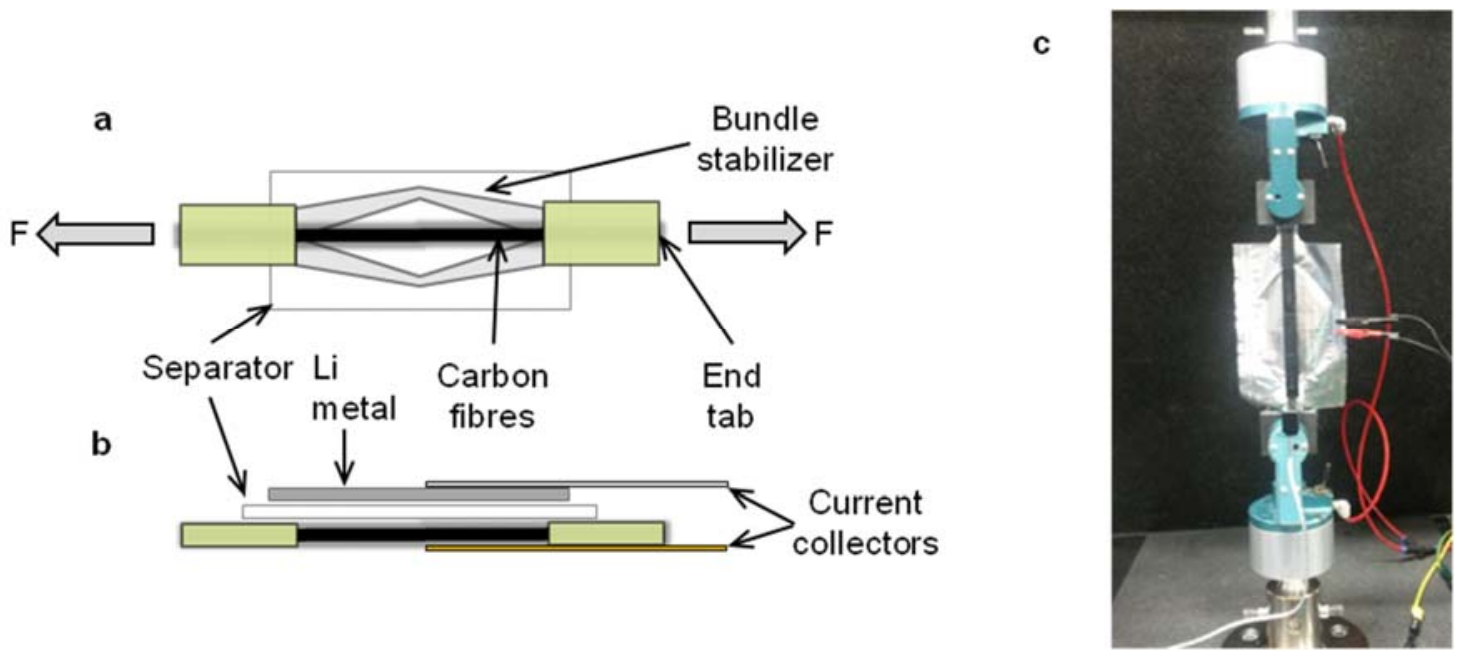

Figure 1. a) The test specimen consisting of a carbon fibre bundle is used as electrode and composite end tabs allow applying an axial mechanical force to the bundle. b) Side-view of the set-up in 'a' to show the current collector and electrode set-up c) Photo of experimental set-up.

The specimens were manufactured from dry carbon fibre bundles according to a method previously described. ${ }^{11}$ The end tabs were about $67 \mathrm{~mm}$ long $\times 18 \mathrm{~mm}$ wide $\times 2 \mathrm{~mm}$ thick. The specimen gauge length, i.e., the length of the carbon fibre bundle between the end tabs was 108 $\mathrm{mm}$. The mass of the carbon fibre electrode facing the Li metal electrode was $20 \mathrm{mg}$ for the T800 bundle and $33.5 \mathrm{mg}$ for the IMS65 bundle. The specimen dimensions were made suitable for the tensile test rig, for the external extensometer and to facilitate the use of the specimens as electrode in electrochemical cells. The Li-ion cell used, as shown in Figure 1a, was manufactured in a glovebox under inert argon atmosphere with less than 1 ppm oxygen and water at ambient temperature. The cell consisted of a carbon fibre working electrode (specimen), a glass microfibre separator (Whatman GF/A, $260 \mu \mathrm{m}$ thick, 90 \% porosity) impregnated with $1200 \mu \mathrm{L}$ of $1.0 \mathrm{M}$ LiPF6 in EC:DEC (1:1 w/w, LP40 Merck) electrolyte and a Li metal counter electrode. Copper and nickel foils were used as current collector tabs. The layup was packed in a vacuum sealed bag (Skultuna Flexible, PET/Al/PE, $12 \mu \mathrm{m} / 9 \mu \mathrm{m} / 75 \mu \mathrm{m}$ thick). The bag was sealed over $15 \mathrm{~mm}$ with sealant tape on the specimen end tabs. Each end tab was partly out of the bag to allow direct gripping of the tester on the specimen and direct measurement of the strain in the carbon fibre 
bundle between the end tabs with an external extensometer (to remove the compliance of the loading system and of the grips).

All cells were subjected to a preliminary characterisation and a couple of electrochemical charging/discharging cycles at a low constant reference current density (13.6 mA/g for the T800 and $15 \mathrm{~mA} / \mathrm{g}$ for the IMS65 specimens, respectively) to be in an electrochemical state which could be considered reproducible.

\section{Experimental setup}

An external extensometer Instron 2620-601 with $162.5 \mathrm{~mm}$ gauge length was used to measure the strain between the end tabs. The mechanical tester was an Instron 5567 equipped with a $5 \mathrm{kN}$ load cell and pneumatic grips which maintain a constant force to the specimen end tabs during long-lasting tests. The cell current collectors were connected to a Solartron 1286 Electrochemical Interface potentiostat controlled with the CorrWare software. Figure 1c shows a finished cell in the grips of the mechanical tester and connected to the potentiostat.

\section{Electrochemical framework}

The Li metal counter electrode can be considered as an infinite source of Li with a constant electrode potential independent of current density and thus also serving as reference electrode in the system. The change in the cell potential thereby corresponds to the change in the carbon fibre electrode potential vs. $\mathrm{Li} / \mathrm{Li}^{+}$. The term lithiation is used to indicate the intercalation (insertion) of $\mathrm{Li}$ in the carbon fibre electrode and delithiation is for the extraction of the same.

The term state of charge (SOC) is used herein to indicate the degree of lithiation of the carbon fibre electrode, i.e., a measure of the amount of Li-ions intercalated in the carbon fibre electrode. Here, fully intercalated corresponds to lithiation at a low constant current density down to 0.002 $\mathrm{V}$ vs. $\mathrm{Li} / \mathrm{Li}^{+}$and a subsequent polarization at constant potential (about $0.030 \mathrm{~V}$ vs. $\mathrm{Li} / \mathrm{Li}^{+}$) to stabilize the cell potential. The $\mathrm{Li}$ in the carbon fibre electrode was extracted at low current density until the electrode reached a potential of $1.50 \mathrm{~V}$ vs. $\mathrm{Li} / \mathrm{Li}^{+}$, and this state was defined as fully delithiated. Thus, the measured fully delithiated and lithiated states were used to define the $0 \%$ and $100 \%$ SOC of the carbon fibre electrode, respectively. At the defined $100 \%$ SOC, the measured capacity was $300 \mathrm{mAh} / \mathrm{g}$ for the T800 cell and $290 \mathrm{mAh} / \mathrm{g}$ for the IMS65 cell.

The change in accessible specific capacity $C_{s}$ during charging or discharging reflects the amount of $\mathrm{Li}$ intercalated or deintercalated in the carbon fibre electrode during the experiment and is calculated from: 


$$
\Delta C_{s}=\int_{0}^{T} I d t / m
$$

which is the electrical current $I$ integrated over the experiment time and $m$ is the mass of carbon fibre electrode.

\section{Measurements of the piezo-electrochemical response}

Applying a mechanical force to Li-intercalated carbon fibres results in a response of the open circuit potential (OCP), i.e., of the carbon fibre electrode potential when no external current is applied. ${ }^{14}$ In order to investigate a possible dependency on the SOC, the OCP response was measured in the delithiated and fully lithiated states (0\% and $100 \%$ SOC), and at intermediate states during lithiation and delithiation of approximately $5 \%, 20 \%, 40 \%, 60 \%$, and $80 \%$ SOC, respectively.

A small tensile extension ( $\sim 0.10 \%$ strain) was applied prior to testing to put the Li-intercalated carbon fibres under tension (the OCP must respond at this initial strain). The OCP response was measured for $0.37 \%(0.40 \mathrm{~mm})$ applied strain at different SOC according to the following procedure. (i) Apply a constant current density until the target electrode potential is reached; (ii) Stabilize the electrode potential to the desired value by applying a constant potential during 5-10 $\mathrm{h}$ or until the absolute current becomes lower than $1 \mu \mathrm{A}$; (iii) Increase the tensile strain of $0.37 \%$, hold for $20 \mathrm{~s}$ and then decrease of the same while measuring the OCP response, repeat twice at two different strain rates $\left(1.85 \times 10^{-4} \mathrm{~s}^{-1}\right.$ and $\left.9.26 \times 10^{-5} \mathrm{~s}^{-1}\right)$.

\section{Energy harvesting experiments}

A battery generally delivers less electrical energy during discharge than provided during charge because of a higher cell potential at charge than at discharge (electrochemical hysteresis), as shown in Figure 2a. This is due to the total cell overpotential, a deviation from equilibrium, i.e. OCP, due to irreversible losses originating from the charge transfer processes, resistances and transport limitations in the battery cell. ${ }^{10}$ The magnitude of these losses depend on the applied current density. However, if the OCP response to a mechanical force carried by the carbon fibre electrode (piezo-electrochemical effect) overtakes the induced overpotential, more electrical energy will be delivered from the cell than provided, as schematically shown in Figure 2b. This can be achieved by adjusting the current density and thereby the overpotential during lithiation and delithiation of the carbon fibre electrode.

The carbon fibre bundles have a higher electrode potential than the Li metal and are in essence used as positive electrodes in our setup. That leads to a higher cell potential during delithiation 
than lithiation of the carbon fibres, opposite to the behaviour of a normal Li-ion battery cell where the carbon material acts as the negative electrode. However, the electrode potential of the counter electrode is not important for demonstration of the concept.
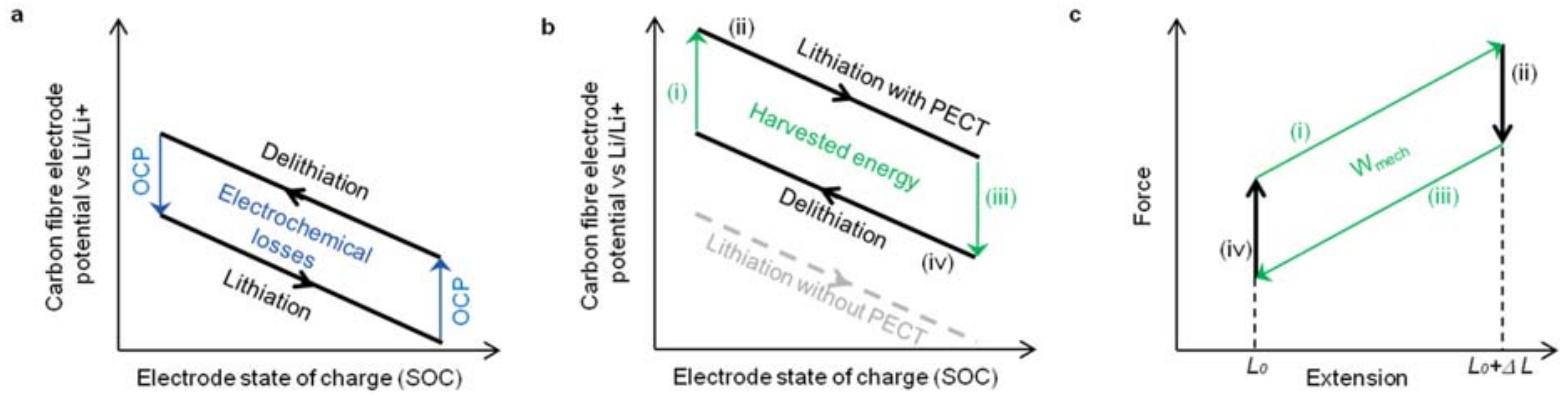

Figure 2. The working electrode (WE) and the counter electrode (CE) of the cell are connected to the potentiostat (P). a) Electrochemical hysteresis when the carbon fibre electrode carries no mechanical force. b) Harvested electrical energy when the carbon fibre electrode subjected to an applied strain $\varepsilon$ and carries a mechanical force $F$ during lithiation c) Mechanical hysteresis perspective of ' $b$ '. Wmech represents the losses in the mechanical work of the external force (see table 1 for explanation of (i) to (iv)).

Table 1 - Description of the energy harvesting procedure and potential response

\begin{tabular}{|l|l|l|l|}
\hline & Electrochemical operation & External strain & Potential vs. lithium \\
\hline (i) & OCP $100 \mathrm{~s}$ & Increasing & Increasing (PECT effect) \\
\hline (ii) & CF lithiation $100 \mathrm{~s}$ & Constant & Decreasing (SOC increase) \\
\hline (iii) & OCP $100 \mathrm{~s}$ & Decreasing & Decreasing (PECT effect) \\
\hline (iv) & CF delithiation $100 \mathrm{~s}$ & Constant & Increasing (SOC decrease) \\
\hline
\end{tabular}

The procedure for harvesting energy is to carry out a 4-step harvesting cycle according to the following route (Figure $2 b-c)$ :

Initial state: From a delithiated state (SOC $0 \%$ ), lithiation at constant current density until a target carbon fibre electrode potential, denoted $E_{0}$, was reached. The potential was stabilized to $E_{0}$ by applying a constant potential for $5 \mathrm{~h}$ or until the absolute current becomes lower than $1 \mu \mathrm{A}$.

Step (i) (OCP): Measure the OCP during $100 \mathrm{~s}$. Increase the applied strain $(1.2 \mathrm{~mm} / \mathrm{min})$ in the specimen $\Delta \varepsilon$ at mid-time, i.e. after about $50 \mathrm{~s}$. The applied strain increases $\Delta \varepsilon=0.37 \%$ which results in an increase of the OCP from $E_{0}$ to $E_{1}$. 
Step (ii) (lithiation): Keep the applied strain constant and run a $100 \mathrm{~s}$ lithiation at a constant current density (cell discharge).

Step (iii) (OCP): Measure the OCP during $100 \mathrm{~s}$. Decrease the applied strain $(1.2 \mathrm{~mm} / \mathrm{min})$ in the specimen $\Delta \varepsilon$ at mid-time, i.e. after about $50 \mathrm{~s}$. When the applied strain decreases $\Delta \varepsilon$, the OCP also decreases to $E_{3}<E_{0}$ since the fibres have been further lithiated during this step.

Step (iv) (delithiation): keep the applied strain constant and run a $100 \mathrm{~s}$ delithiation (cell charge) at the same constant current density as for the lithiation. The final cell potential is then back to $E_{0}$ since the applied strain and state of charge are the same as before step (i).

\section{RESULTS AND DISCUSSION}

\section{Piezo-electrochemical response}

Figure 3 shows the OCP response to an external force for an applied strain of $0.37 \%$ and for two strain rates of a T800 specimen. The response clearly shows a change in the potential of the Li-intercalated carbon-fibre electrode induced by the mechanical force. It appeared to be fast, linear and reversible with the applied strain, and the same for two applied strain rates which supports that it might be used for strain sensing as already stated. ${ }^{14}$ Figure 4 plots the OCP response as function of the carbon fibre SOC for both T800 and IMS65 specimens. The response appears to reach a maximum of about $5.4 \mathrm{mV}$ at $\sim 60 \%$ SOC for the T800 specimen. For the IMS specimens the values are slightly lower, about $3.6 \mathrm{mV}$. For a fully deintercalated carbon fibre (0 \% SOC), it was still possible to measure a fairly high OCP response ( $\sim 4 \mathrm{mV}$ for T800 and $\sim 3 \mathrm{mV}$ for IMS65) which is attributed to that some Li still remains intercalated (trapped) in the delithiated fibres. ${ }^{12}$ The overall variation in the OCP response with the SOC was limited to about $30 \%$ implying that energy harvesting can be performed at any initial SOC. 


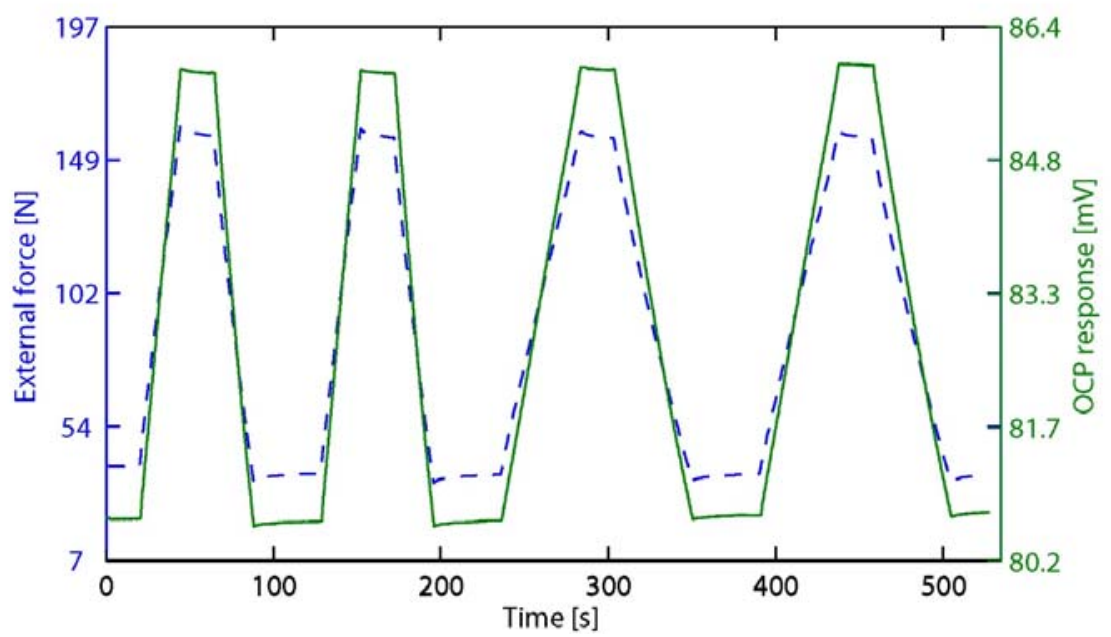

Figure 3. OCP response (solid line) to an external force (dashed line) at about $80 \%$ SOC and for an applied strain of $0.37 \%$.

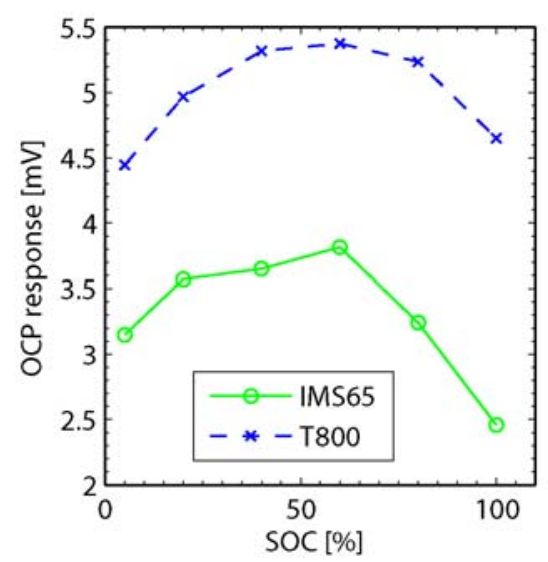

Figure 4. Measured piezo-electrochemical response of T800 specimen and IMS65 specimen as function of SOC during lithiation for an applied strain of $0.37 \%$.

\section{Harvested energy and power}

The specific electrical energy harvested per cycle $W_{\text {elec }}$ is the difference between the electrical work delivered during lithiation (cell discharge) and the electrical work provided during delithiation (cell charge) of the carbon fibre electrode (the area of the encircled charge/discharge cycles shown in Figure 5):

$$
W_{\text {elec }}=I \times \int_{0}^{T} E_{l i} d t / m-I \times \int_{0}^{T} E_{\text {deli }} d t / m
$$


where $E_{l i}$ and $E_{\text {deli }}$ are the cell potentials during lithation and delithiation, respectively, $I$ is the absolute value of the applied current, $T$ is the duration of the galvanostatic steps, i.e. $100 \mathrm{~s}$, and $m$ is the mass of carbon fibre electrode. The harvester specific power is the useful energy per cycle divided by the cycle time. The specific energy per cycle and the specific power were measured for different current densities at electrode potentials of about $5 \%, 20 \%, 50 \%$ and $80 \%$ SOC. The overpotential still induces a voltage drop between OCP and lithiation so that $\Delta E_{l i}<0$ and a voltage increase between OCP and delithiation $\Delta E_{\text {deli }}>0$ (see Figure $5 a$ ). The specific electrical energy harvested for an ideal harvesting cycle (without electrochemical losses) could be estimated through:

$$
W_{\text {elec, }, \text { deal }}=I \times \int_{0}^{T}\left[\left(E_{l i}-\Delta E_{l i}\right)-\left(E_{\text {deli }}-\Delta E_{\text {deli }}\right)\right] d t / m
$$

Figure 5a-d shows energy harvesting cycles at $0.6 \mathrm{~mA} / \mathrm{g}$ and $1.2 \mathrm{~mA} / \mathrm{g}$ for increasing SOCs for T800 specimens. Each step of the harvesting cycle is numbered and oriented with an arrow at $6 \%$ SOC and $0.6 \mathrm{~mA} / \mathrm{g}$ for clarity. For each cycle, step (i) is the left vertical line, step (ii) is the top slightly decreasing horizontal curve, step (iii) is the right vertical line and step (iv) is the bottom slightly increasing horizontal curve. Lithiation of the carbon fibre electrode was consistently performed at higher potential than delithiation which clearly demonstrates the concept of piezoelectrochemical energy harvesting. As denoted in Eq. 3, at the end of step (i) and as step (ii) is started a voltage drop of $\Delta E_{l i}<0$ is seen (top left corner, indicated with arrow in Figure $5 a$ ). Similarly, at the end of step (iii) and as step (iv) is started a voltage increase of $\Delta E_{d e l i}>0$ is seen (bottom right corner). This electrochemical overpotential is expectedly higher at higher current density, and is found to depend on the SOC and to be for instance higher at $81 \%$ SOC than at 24 $\%$ SOC.
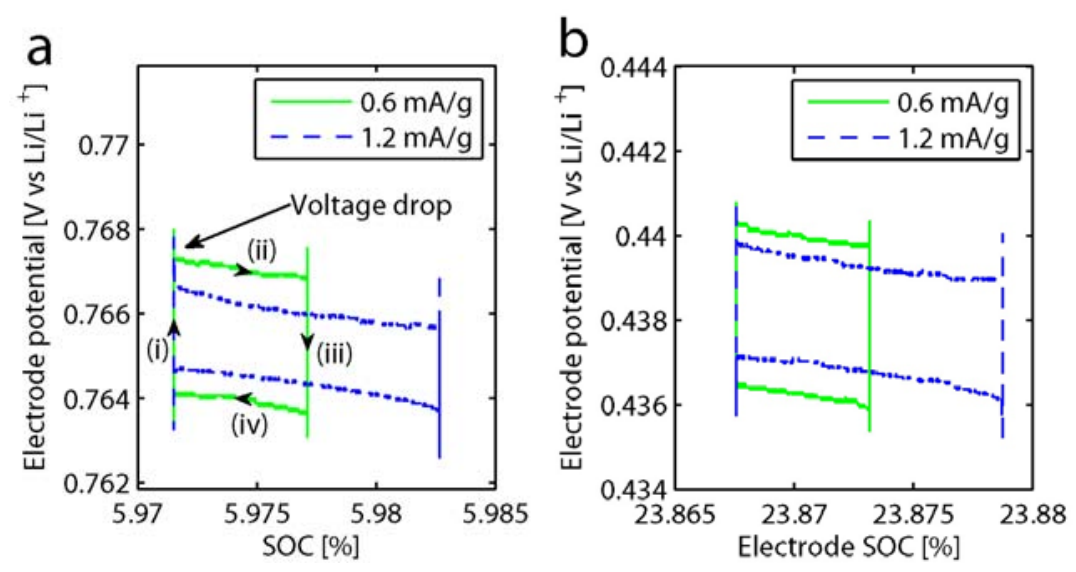

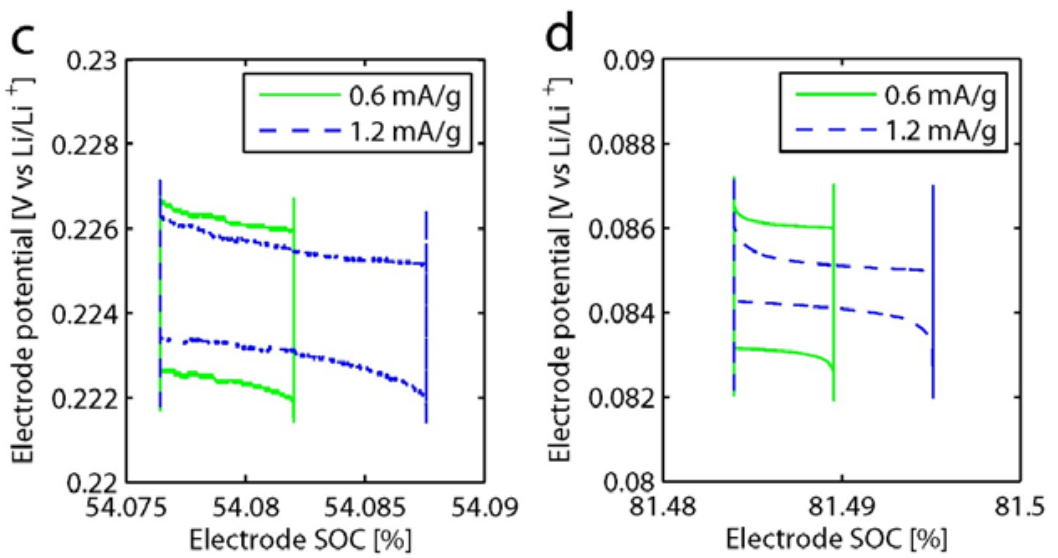

Figure 5a-d. The results of the measured piezo-electrochemical harvesting cycles are shown for two different current densities at four different SOCs.

The harvester specific electrical power is shown in Figure 6a and 6b for T800 and IMS65, respectively. Depending on the SOC, the current density below which it was possible to perform a harvesting cycle with T800 was 1.2-2.5 mA/g (dashed line) and the specific power reaches a maximum at $0.8-1.2 \mathrm{~mA} / \mathrm{g}$. For IMS65 the corresponding values were $0.6-1.2 \mathrm{~mA} / \mathrm{g}$ and $0.4-0.6$ $\mathrm{mA} / \mathrm{g}$. The specific power is expectedly lower at the lowest current densities because the change in SOC is smaller during the galvanostatic steps, see Equation (3). At higher current densities the specific power is also expectedly smaller because the overpotential increases. A maximum of 0.85 $\mu \mathrm{W} / \mathrm{g}$ was measured for the T800 specimen at about $20 \%$ SOC for a current density of $1.2 \mathrm{~mA} / \mathrm{g}$. At $5 \%$ and $80 \%$ SOC the specific power was lower. The measured maximum for IMS65 was of about $0.30 \mu \mathrm{W} / \mathrm{g}$ for a current density of $0.6 \mathrm{~mA} / \mathrm{g}$ at about $20 \%$ SOC. The specific power was lower and had a maximum at lower current densities for the IMS65 bundle which may be due to the material or an effect of a thicker bundle. The measured piezo-electrochemical responses were also smaller for IMS65 as seen in Figure 4. 

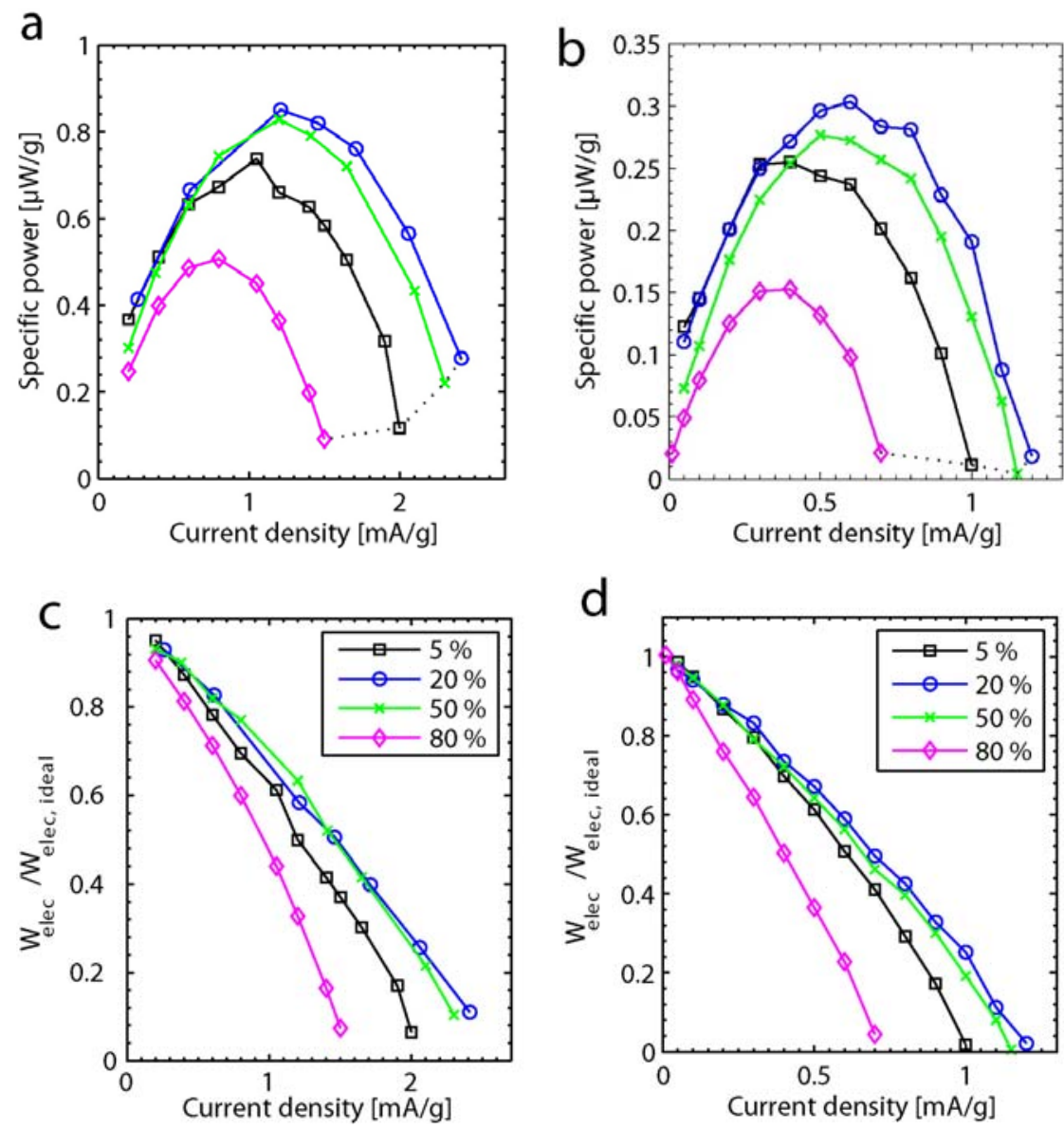

Figure 6. Specific electrical power and electrochemical efficiency of the harvester as function of the current density with (a) and (c) the T800 and (b) and (d) the IMS65 specimens. The results are at SOCs of about $5 \%$ (square marker), $20 \%$ (circle marker), $50 \%$ (x-marker), and $80 \%$ (diamond marker) for the T800H and IMS65 specimens, respectively.

The electrochemical efficiency as function of the current density used during the harvesting cycles is shown in Figure 6c and d as the ratio $W_{\text {elec }} / W_{\text {elec,ideal }}$ for the different SOCs (see Eq. 2 and 3). This ratio approaches 1 when the overpotential becomes negligible. The efficiency was then expectedly higher at lower current densities, and also at the intermediate SOCs. Thus, the harvested specific power, the ratio $W_{\text {elec }} / W_{\text {elec,ideal }}$ and the OCP response to an applied strain exhibited similar trends as function of SOC and reached a maximum at intermediate SOC which appeared to be the most favourable for piezo-electrochemical energy harvesting. At the current density for which the specific power is at maximum the ratio $W_{\text {elec }} / W_{\text {elec,ideal }}$ appears to be about 0.6-0.7 and similar at all SOCs. Considering a density of $7.5 \mathrm{~g} / \mathrm{cm}^{3}$, the typical specific power of 
MEMS PZT piezoelectric energy harvesters are in the order of $100 \mu \mathrm{W} / \mathrm{g} .^{2}$ However, the frequencies are typically $100-1,000 \mathrm{~Hz}$ which leads to a specific energy per cycle in the order of 0.1-1 $\mu \mathrm{J} / \mathrm{g}$ per cycle. The specific energy per cycle of the present harvester is much higher, 100$350 \mu \mathrm{J} / \mathrm{g}$ per cycle at a frequency of $2.5 \mathrm{mHz}$ (for $400 \mathrm{~s}$ cycle time), and it could be even higher for higher cycle times. Thus, from this perspective it seems most advantages to use the piezoelectrochemical effect to harvest energy from mechanical motions of low frequencies.

\section{Losses in mechanical work}

Reversible Li-intercalation expansion strains will cause the force carried by the carbon fibre electrode (subjected to a constant strain) to decrease during lithiation (step (ii)) and increase during delithiation (step (iv) $)^{12}$ resulting in a mechanical hysteresis. The electrical energy is harvested from this mechanical hysteresis, i.e., from the losses in the mechanical work of the external force induced by reversible Li-intercalation expansion strain during the harvesting cycle. Figure 2c is a schematic of the external mechanical force as function of the applied extension during a 4-step harvesting cycle. During lithiation (step (ii)), the carbon fibres expand causing a drop in the external mechanical force ${ }^{12}$ which leads to that the mechanical work during the strain release (step (iii)) is smaller than during application of the strain (step (i)). The mechanical hysteresis $W_{\text {mech }}$ corresponds to the difference between the mechanical work of the external applied force when the strain is increased (during step (i)) and when it is decreased (during step (iii)):

$$
W_{\text {mech }}=\Delta f \times \Delta L / m
$$

where $\Delta f$ is the estimated change in the external force during a lithiation or delithiation step at constant applied strain (step (ii) in Figure 2c), $\Delta L$ is the change in the applied extension during the OCP steps ((i) and (iii)), and $m$ is the mass of carbon fibre electrode.

\section{Harvesting efficiency}

The loss in the mechanical work during a harvesting cycle was of course expected to be at least as large, or larger than the harvested electrical energy. However, in the present work, only very low amount of $\mathrm{Li}(\sim 0.01 \mathrm{mAh} / \mathrm{g})$ is intercalated during the energy harvesting cycle in step (ii) (due to low charging time and rates) and the measured mechanical hysteresis was very small and not measureable with any acceptable accuracy. Therefore, the loss in mechanical work $W_{\text {mech }}$ was instead estimated using following procedure; Starting at a low SOC, about $5 \%$ SOC, the specimens were subjected to a constant mechanical strain of $\sim 0.5 \%$. The specimen was thereafter subjected to a lithiation at constant current density causing a longitudinal expansion of the carbon fibre, and 
the subsequent load drop was measured as function of SOC using the same procedure as in. ${ }^{12}$ The same low current densities were used as in the harvesting cycles during a time long enough to intercalate about 5-10 mAh/g in order to more accurately measure the load drop. These experimental results allowed for the estimation of the force drop during the lithiation at a constant current density (step (ii)) of the harvesting cycles, i.e., of $W_{\text {mech }}$ at different current densities using Eq. 4 assuming that the carbon fibre bundle has constant stiffness. ${ }^{13}$

Figure 7 plots the ratios between the harvested electrical energy and the mechanical losses at $5 \%$ SOC. For lower current densities, the ratio approaches one which indicates small electrochemical and transduction losses. However, due to variations and errors in the $W_{\text {mech }}$ estimates, the calculation of energy ratios becomes very inaccurate at the lowest current densities used. At higher current densities, the electrochemical as well as the transduction losses expectedly increase.

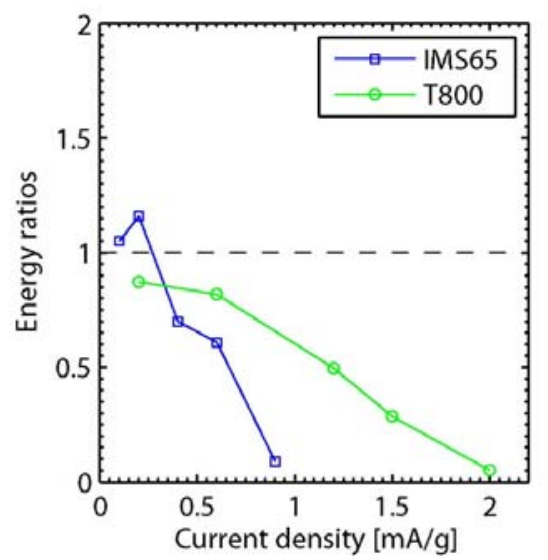

Figure 7. Electrical-to-mechanical energy ratios of the T800 specimens (circle marker) and the IMS65 specimens (square marker) as function of the current density used during the harvesting cycles at about $5 \%$ SOC.

\section{Paths for improved harvesting efficiency}

There are several paths to significantly increase the harvesting frequency and power density. First of all, steps (i) and (iii) in the present cycle could be cut to only a few seconds resulting in the same energy per cycle but thus almost twice the output power. We have used carbon fibre bundles herein but as shown previously ${ }^{10}$ the overpotentials can be decreased and the rate of lithiation increased significantly when using single fibres. By using e.g. spread fibre bundles one can increase the current density in steps (ii) and (iv) while still maintaining low overpotentials 
which again would increase the harvested power significantly. Yet another way to increase the power is to increase the applied strain on the fibre which increases the OCP response, however, this is of course limited by the failure strain of the carbon fibres which is in the order of $1.5-2 \%$. All these things together might make it possible to perform energy harvesting with significantly higher power density using this device in the range of $1 \mathrm{~Hz}$ in the future.

\section{CONCLUSIONS}

A proof of concept showing that a piezo-electrochemical effect in carbon fibres can be used for energy harvesting has been shown for the first time. A reversible response of Li-intercalated carbon fibre electrode potential to an applied mechanical strain was measured for a range of SOCs. At low current densities, typically lower than $0.5-2 \mathrm{~mA} / \mathrm{g}$, it was possible to discharge the cell at higher cell potential than at charge, harvesting energy from the applied external mechanical work. At intermediate SOC, the specific power harvested reached a maximum which was in the order of 0.3-1 $\mu \mathrm{W} / \mathrm{g}$ for current densities of about $0.4-1.2 \mathrm{~mA} / \mathrm{g}$ at an operating frequency in the order of $\mathrm{mHz}$. The energy per cycle was particularly promising and appeared to be in the order of 100 times more than for PZT piezoceramics harvesters in the present tests. There are several promising paths to increase the power output of this new type of energy harvesting concept.

This work shows a completely new path for energy harvesting using Li intercalation in carbon fibres which appears to be general to this type of material. It could open up for a new field of research possibly involving other ion intercalating materials and electrochemical processes. With the development of structural polymer electrolytes that can provide stiffness and strength one can envisage carbon fibre composite materials with both structural and energy storage capability ${ }^{18}$ and that such materials also can be used for converting mechanical energy, e.g. bending, vibrations, etc., to electrical energy.

Since this approach is based on the concept for Li-ion battery technology the same device can also be used for storing energy by just adding an appropriate counter electrode, forming a true Liion battery cell. One vision of this work is to construct a material combination that can harvest mechanical energy and store it in the same device, i.e., make a battery that can be charged using external alternating mechanical loads. 


\section{AUTHOR INFORMATION}

\section{Corresponding Author}

* Fax: +46 8 108087. E-mail: gnli@kth.se

\section{Notes}

The authors declare no competing financial interest.

\section{ACKNOWLEDGMENT}

We thank the Swedish Research Council, projects 621-2012-3764 and 621-2014-4577, the Swedish Energy Agency, project 37712-1 and the strategic innovation programme LIGHTer (provided by Vinnova, the Swedish Energy Agency and Formas) for financial support. The Swedish research group Kombatt is acknowledged for its synergism throughout this work.

\section{REFERENCES}

(1) Kim, H. S.; Kim, J.-H.; Kim, J. A Review of Piezoelectric Energy Harvesting based on Vibration. Int. J. Precision Engineering and Manufacturing. 2011, 12, 1129-1141.

(2) Radousky, H. B.; Liang, H. Energy Harvesting: An Integrated View of Materials, Devices and Applications. Nanotechnology. 2012, 23, 1-35.

(3) Kim, S-G.; Priya, S.; Kanno, I. Piezoelectric MEMS for Energy Harvesting. MRS Bull. 2012, 37, 1039-1050.

(4) Lee, S. W.; Yang, Y.; Lee, H.-W.; Ghasemi, H.; Kraemer, D.; Chen, G. An Electrochemical System for Efficiently Harvesting Low-Grade Heat Energy. Nat. Commun. 2013, 5, 1-6, Article number 3942.

(5) Mitcheson, D.; Yeatman, E. M.; Kondala Rao, G. K.; Holmes, A. S.; Green, T. C. Energy Harvesting from Human and Machine Motion for Wireless Electronic Devices. Proc. IEEE. 2008, 96, 1457-1486.

(6) Priya, S. Advances in Energy Harvesting using Low Profile Piezoelectric Transducers. $J$. Electroceram. 2007, 19, 165-182. 
(7) Byoungwoo, K.; Gerbrand, C. Battery Materials for Ultrafast Charging and Discharging. Nature. 2009, 458, 190-193.

(8) Snyder, J. F.; Wong, E. L.; Hubbard, C. W. Evaluation of Commercially Available Carbon Fibres, Fabrics, and Papers for Potential use in Multifunctional Energy Storage Applications. $J$. Electrochem. Soc. 2009, 156, A215-A224.

(9) Kjell, M. H.; Jacques, E.; Zenkert, D.; Behm, M.; Lindbergh, G. PAN-based Carbon Fiber Negative Electrodes for Structural Lithium-Ion Batteries. J. Electrochem. Soc. 2001, 158, A1455A1460.

(10) Kjell, M. H.; Zavalis, T. G.; Behm, M.; Lindbergh, G. Characterization of Lithium Intercalation Processes of PAN-based Carbon Fibres in a Microelectrode System. J. Electrochem. Soc. 2013, 160, A1473-A1481.

(11) Jacques, E.; Kjell, M. H.; Zenkert, D.; Lindbergh, G.; Behm, M.; Willgert, M. Impact of Electrochemical Cycling on the Tensile Properties of Carbon Fibres for Structural Lithium-Ion Composite Batteries. Compos. Sci. Techol. 2012, 72, 792-798.

(12) Jacques, E.; Kjell, M. H.; Zenkert, D.; Lindbergh, G.; Behm, M. Expansion of Carbon Fibres induced by Lithium Intercalation for Structural Electrode Applications. Carbon. 2013, 59, 246-254.

(13) Jacques, E.; Kjell, M. H.; Zenkert, D.; Lindbergh, G. The Effect of Lithium-Intercalation on the Mechanical Properties of Carbon Fibres. Carbon. 2014, 68, 725-733.

(14) Jacques, E.; Kjell, M. H.; Zenkert, D.; Lindbergh, G. Piezo-Electrochemical Effect in Lithium-Intercalated Carbon Fibres. Electrochem. Commun. 2013, 35, 65-67.

(15) Koyama Y.; Chin T. E.; Rhyner U.; Holman R. K.; Hall S. R.; Chiang Y.-M. Harnessing the Actuation Potential of Solid-State Intercalation Compounds. Adv. Funct. Mater., 2006, 16, 492-498.

(16) Chin T. E.; Rhyner U.; Koyama Y.; Hall S. R.; Chiang Y.-M. Lithium Rechargeable Batteries as Electromechanical Actuators. Electrochem. Solid-State Lett., 2006, 9, A134-A138. 
(17) Kading G. A. Piezo-Electrochemical Transducer Effect (PECT). PhD thesis, Air Force Institute of Technology, Ohio, USA, 2007.

(18) Asp, L., and Greenhalgh, E., Structural Power Composites. Compos. Sci. Techol. 2014, $101,41-61$.

(19) Xue X., Wang S., Guo W., Zhang Y. and Wang Z.L., Hybridizing Energy Conversion and Storage in a Mechanical-to-Electrochemical Process for Self-Charging Power Cell. Nano Lett., 2012, 12, 5048-54.

(20) Zhang ,Y., Zhang Y., Xue X., Cui C., He B., Nie Y., Deng P. and Wang Z.L., PVDF-PZT Nanocomposite Film based Self-Charging Power Cell. Nanotechnology. 2014, 25, 105401 